\title{
Fifteen Years Post Aortic Root Reconstruction of a Giant Ascending Aortic Aneurysm
}

\author{
Khaled E. Al-Ebrahim, FRCSC \\ Department of Cardiothoracic Surgery, King Abdulaziz University, Jeddah, Saudi Arabia
}

\section{ABSTRACT}

This is a report of successful repair of a huge ascending aortic aneurysm of $13 \mathrm{~cm}$; it is the biggest aneurysm ever reported. Aortic root replacement using a composite graft and coronary implantation was done. There was dramatic improvement in ejection fraction and renal function post repair. This patient has been followed up on for 15 years with no complications and leads a relatively normal life.

\section{CASE HISTORY}

A 55-year-old Indonesian male presented to our institution in 2005, with extreme shortness of breath. He was tachycardic, and there was a visible, forceful apex beat and bilateral crepitations. Blood urea nitrogen and creatinine were elevated. Electrocardiography and chest x-ray showed left ventricular dilatation and pulmonary congestion. Echocardiography revealed severe aortic regurgitation and poor left ventricular function of $20 \%$ ejection fraction. Computerized tomography (CT) of the chest revealed a $13 \mathrm{~cm}$-wide ascending aortic aneurysm limited to the ascending aorta. (Figures 1, 2, and 3) Intraoperatively, cardiopulmonary bypass was established using ascending aorta and bicaval right atrial cannulation. Left ventricular venting was achieved using the right superior pulmonary vein. The heart was arrested using retrograde coronary sinus cardioplegia.

The aneurysm involved the sinus of Valsalva, causing cephalad displacement of the coronary ostia. A size 31 composite graft was sewn to the aortic root and distal ascending aorta. The left and right coronary ostia buttons were trimmed and anastomosed to the appropriate site in the Dacron graft. Postoperative course was smooth, and the patient was discharged after achieving proper anticoagulation levels. Two months postoperatively, the patient complained of progressive dyspnea. Echocardiography showed large hemorrhagic pericardial effusion compressing the right side, which was evacuated using a small anterior right thoracotomy. No other complications occurred, and the patient regularly has followed up in the clinic maintaining his INR 2-3 for more than

Received December 23, 2019; accepted Fanuary 30, 2020.

Correspondence: Khaled E. Al-Ebrabim, Department of Surgery, King Abdul Aziz University, P.O. Box 80215, Feddah 21589, Saudi Arabia; fax: +6401238; +6401000 (e-mail: Dr.k.ebrabim@gmail.com).

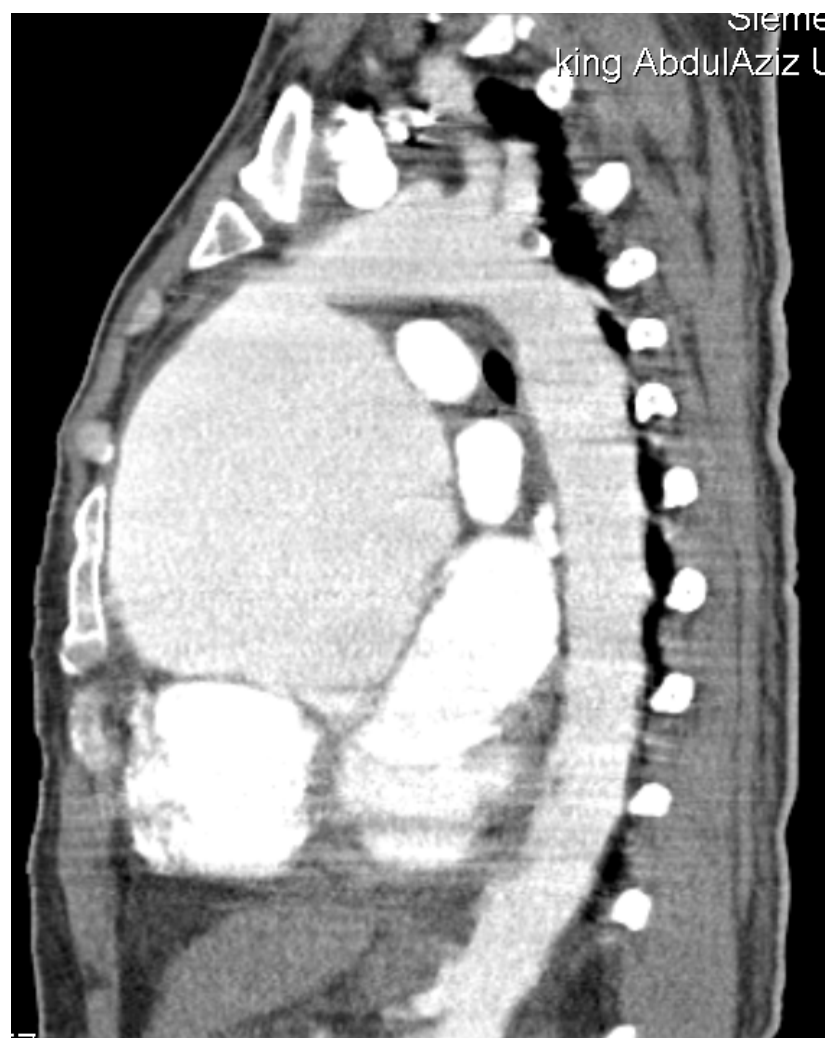

Figure 1. Preoperative sagittal section CT showing the aneurysm.

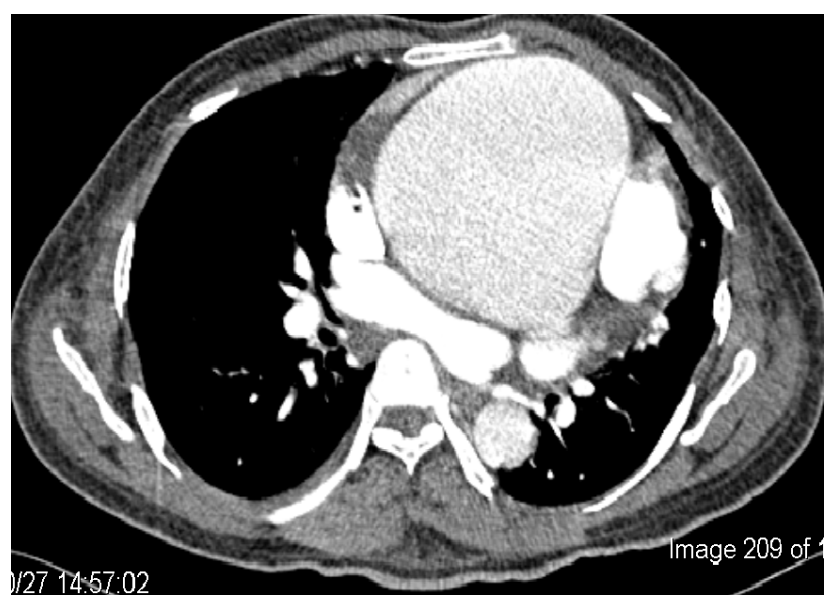

Figure 2. Preoperative axial section CT showing the aneurysm. 


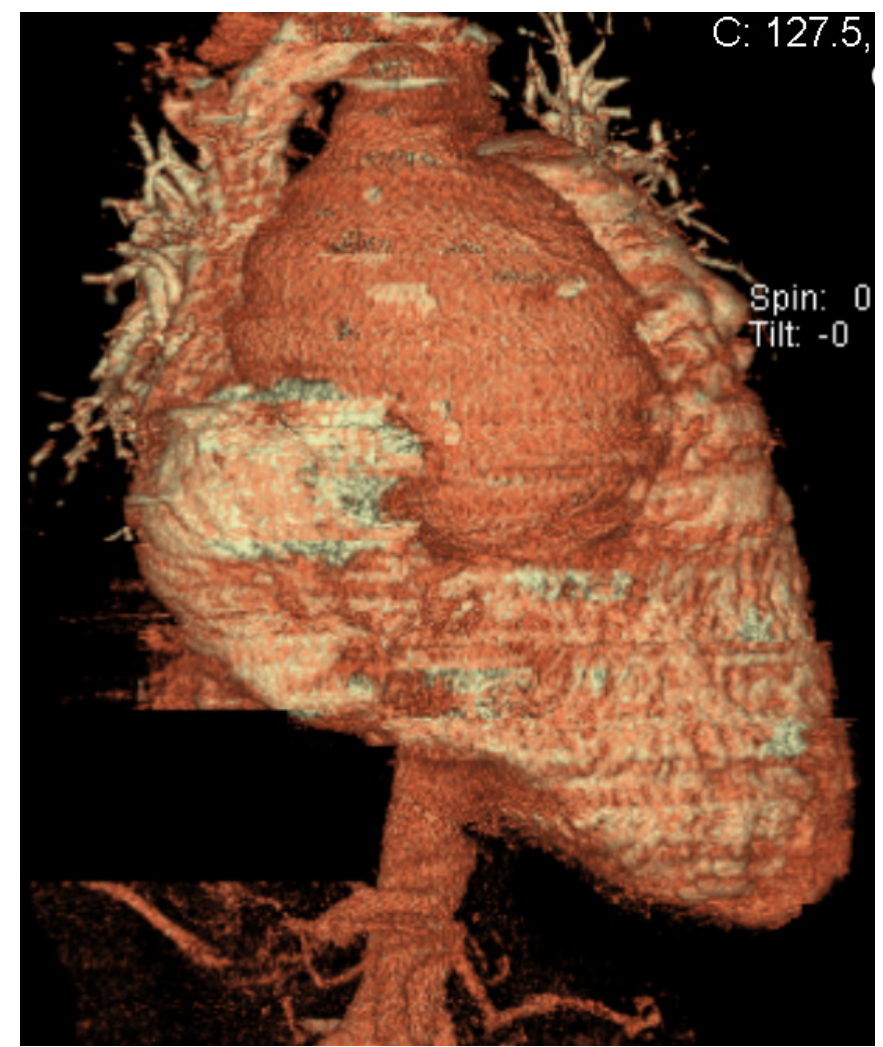

Figure 3. Preoperative 3D CT showing the aneurysm.

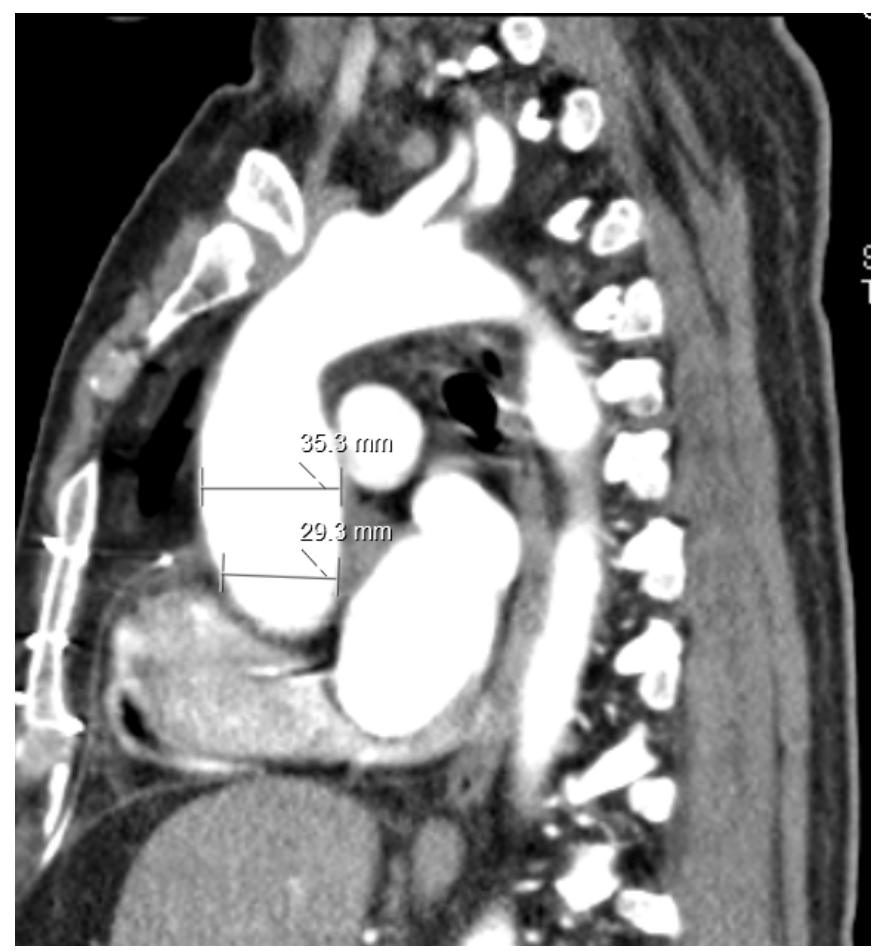

Figure 4. Postoperative axial section showing the measurements of aortic root and ascending aorta.
15 years. His renal function normalized and left ventricular ejection fraction improved to $55 \%$ with normal valve function and without thromboembolic episodes. Follow-up CT was done (Figures 4 and 5).

\section{DISCUSSION}

This is the biggest ascending aortic aneurysm reported in literature. The aortic root transverse diameter was $13 \mathrm{~cm}$ intraoperatively and by CT. The aneurysm actually was bigger than the heart size. This aneurysm caused heart failure and low cardiac output in this patient, leading to renal impairment. Other complications of aneurysms include dissection, compression of adjacent structures - mainly the superior vena cava, innominate veins, right pulmonary artery, trachea, bronchi, and sternal erosion. This aneurysm was atherosclerotic and non-necrotizing with no evidence of dissection or inflammation. Connective tissue disorder is the most common non-infective etiology of aortic root pathology. Marfan's syndrome, Ehlers-Danlos syndrome and Loeys-Dietz syndrome predominantly involve the elastic layer of the aortic root [Cannata 2008]. Screening for giant cell arteritis, Takayasu's arteritis, Kawazaki's disease, syphilis and cocaine use is helpful to reach a diagnosis [Lange 2001; Al Ebrahim 1998].

Composite graft replacement of the aortic valve and

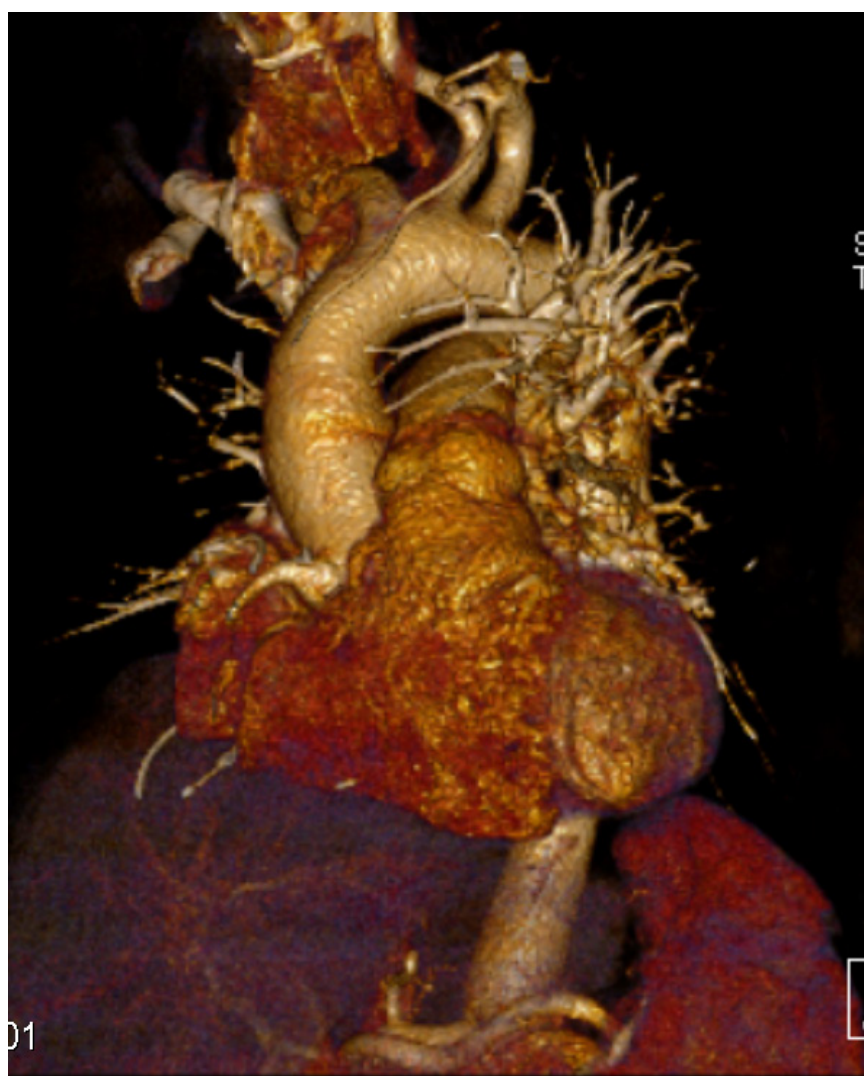

Figure 5. Postoperative 3D CT showing the aortic graft and the reimplanted right coronary artery. 
ascending aorta with reimplantation of the coronary arteries remains the standard operation for patients with aortic root aneurysm. First aortic root replacement was reported by Bentall and De Bono in 1968. Following new technical advances like aortic root reimplantation (David procedure) and remodeling (Yacoub operation) with multiple contemporary modifications, aortic root reconstruction now is widely used in treatment of chronic aortic aneurysmal disease and acute aortic dissections [Cattaneo 2004; Bentall 1968; David 2010; Yacoub 1998]. Either ready-made composite graft is used or intraoperative tailoring can be done if the proper size is not available. The aneurysm was close to the back of the sternum, necessitating careful sternotomy. The neck of the aneurysm was just proximal to the aortic arch branching and thus facilitating central cannulation and clamping.

According to the American Association of Thoracic Surgeons, surgical repair should be performed when the aortic diameter (root or ascending aorta) is $55 \mathrm{~mm}$ or more in patients without risk factors, $50 \mathrm{~mm}$ or more in patients with bicuspid aortic valve, and $45 \mathrm{~mm}$ or more in patients undergoing concomitant other cardiac surgery [Yuan 2011].

The aortic valve could not be spared in this patient as there was huge annular dilatation of about $60 \mathrm{~mm}$, causing a major size discrepancy between the graft and aortic root. This patient had gradual improvement in renal and cardiac function. He regularly is screened for pseudoaneurysm formation or bleeding. Over the past 15 years, there have been no thromboembolic or infective episodes. Surgical skills, use of improved graft substitutes, and local hemostatic agents have made this procedure safer. Results have been good in centers that perform these procedures in high volume. Aortic root replacement with cryopreserved homograft tissue often is used for patients with an infected aortic root.

\section{ACKNOWLEDGEMENT}

This project was funded by the Deanship of Scientific Research (DSR), King Abdulaziz University, Jeddah, Saudi Arabia, under grant No. (1441-66-140) . The authors therefore, gratefully acknowledge the DSR technical and financial support.

\section{REFERENCES}

Al Ebrahim KE. 1998. Syphilitic Aneurysm Revisited. Journal of King Abdulaziz University - Medical Sciences 6:63-66.

Bentall H., DeBono A. 1968. A technique for complete replacement of the ascending aorta. Thorax 23:338-339.

Cannata A, Russo CF, Vitali E. 2008. Bicuspid aortic valve: About natural history of ascending aorta aneurysms. The Annals of Thoracic Surgery 85(1):362-363.

Cattaneo SM, Bethea BT, Alejo DE, Spevak PJ, Clauss SB, Dietz HC, Gott VL, Cameron DE. 2004. Surgery for aortic aneurysm in children: a 21-year experience in 20 patients. Ann Thorac Surg 77: 168-176.

David TE, Maganti M, Armstrong S. 2010. Aortic root aneurysm: Principles of repair and long-term follow-up. The Journal of Thoracic and Cardiovascular Surgery. 140(6 Suppl):S14-S19 discussion S45-51.

Lange RA, Hillis LD. 2001. Cardiovascular complications of cocaine use. N Engl J Med. 2:345-351.

Yacoub M.H., Gehle P, Chandrasekaran V, Burks ES, Child A, RadleySmith R. 1998. Late results of a valve-preserving operation in patients with aneurysm of the ascending aorta and root. J Thorac Cardiovasc Surg 115:1080-109.

Yuan SM, Jing H. 2011. Cystic medial necrosis: Pathological findings and clinical implications. Revista Brasileira de Cirurgia Cardiovascular. 26(1):107-115. 